\title{
Ultraviolet Absorption-Line Studies of the Gaseous Component in Planetary Nebulae
}

\author{
Harriet L. Dinerstein ${ }^{1}$ \\ ${ }^{1}$ University of Texas, Department of Astronomy, 1 University Station, C1400, Austin, TX, \\ $78712-0259$ \\ email: harriet@astro.as.utexas.edu
}

\begin{abstract}
The physical and abundance properties of the gaseous components of planetary nebulae have traditionally been studied using their rich, pan-chromatic emission line spectra. However, absorption lines produced within the column of nebular gas in front of the central star can provide complementary information, giving access to ions and energy levels that cannot be studied via emission lines and yielding new evidence on the presence and nature of internal inhomogeneities. The far ultraviolet (UV) spectral region, as studied with HST and FUSE, is particularly useful for probing species in the photodissociation region (PDR), such as $\mathrm{H}_{2}$ and $\mathrm{O}$. . Comparison of absorption-line and emission line results within the same nebula indicates strong spatial segregation of the molecular material - and possibly of the dust as well - globally and/or on small physical scales. Measurements of UV absorption lines from excited fine-structure levels of $\mathrm{O}$ I reveal that non-thermal (e.g. fluorescent) processes can have an important influence on the level populations, affecting common interpretations of the important infrared cooling lines at 63 and $145 \mu \mathrm{m}$. Unsaturated absorption lines usable for deriving column densities and their ratios can be seen from excited fine-structure levels of some ions abundant in the ionized zone, and in some cases from ions of rarer elements such as the neutron-capture element Ge, which can be self-enriched due to nuclear processing in the PN progenitor star.
\end{abstract}

Keywords. Planetary Nebulae: general—Techniques: spectroscopic—ultraviolet: ISM

\section{Introduction: Absorption vs Emission Line Methods}

Nearly all of our knowledge about the physical conditions and chemical composition of planetary nebulae $(\mathrm{PNe})$ has been obtained from their emission spectra, in particular their rich array of atomic, ionic, and molecular emission lines. The emission lines are sensitive to and carry detailed information about properties such as gas density, kinetic temperature, ionization equilibrium, inhomogeneities, and elemental abundances. So why use absorption lines? Absorption lines suffer from various limitations and potential problems in interpretation, but they also provide a novel perspective on nebular properties. I begin this review by comparing the properties of emission and absorption lines, highlighting their respective advantages and disadvantages.

By their nature, emission lines directly trace only the fraction of a species in the upper level of the transition. For optical and UV emission lines (which generally arise from excited terms of the ground configuration), the excitation energy is large relative to the mean kinetic energy of the thermal electrons, $\chi \geqslant k T_{e}$ ) and the population fraction is small, $\leqslant 10^{-3}$. On the other hand, excited fine-structure levels of the ground term, which give rise to infrared lines, can contain large fractions of their respective ions and are relatively insensitive to $T_{e}$ (e.g. Dinerstein 1995). Derivation of total ionic abundances requires calculation of the equilibrium among lowest few energy levels, but as long as the assumptions (e.g. collisional excitation only) are valid and the physical conditions well known, such ionic abundances are usually regarded as fairly reliable. 
The presence of a bound electron in an excited level is not a prerequisite for producing absorption lines; indeed, the strongest lines are generally from the ground levels of each ion. Since the photons originate from the central star, there is no selection against short-wavelength lines, even when $h \nu \ggg k T$. The strongest resonance lines of most abundant neutral and ionized species lie in the UV, making it the optimal spectral region for absorption-line studies. The intrinsic strengths of these lines are advantageous for detecting low column densities, e.g. rare ions, or excited levels, but are a disadvantage for more abundant species, leading to line saturation. Saturated lines are unsuitable for measuring column densities, become broadened, and often blend with other velocity components of the same line or with transitions of other species.

Another attribute of absorption-line studies is that high spectral resolution is required in order to resolve nebular from interstellar (IS) velocity components, particularly for transitions that may arise in both locations. This effectively restricts nebular absorptionline studies to sources with large peculiar velocities, in order to minimize confusion and blending. On the other hand, such high spectral-resolution observations also provide information about the gas kinematics through Doppler shifts and line profiles.

Finally, the application of the absorption-line method introduces unavoidable selection effects. It requires a background continuum source, in this case the central star. Since the star is effectively a point source, absorption lines sample only a narrow pencil beam through the circumstellar envelope. Emission lines integrate over a larger volume, so if inhomogeneities are present, they sample a range of conditions. Furthermore, it is impractical to measure UV absorption lines if the line of sight extinction, $A_{V}$, is large, because the continuum will be too faint. Thus, UV absorption-line studies tend to be successful only for lines of sight with low (and/or dust-deficient) overall column densities.

\section{Facilities for UV Absorption-Line Spectroscopy: A Brief History}

The pioneering space instrument for high-resolution UV spectroscopy, deployed in the early 1970's, was Copernicus (Spitzer \& Jenkins 1975). Because its spectral coverage extended down to $950 \AA$, Copernicus was able to directly observe absorption lines from the ground electronic and vibrational state of $\mathrm{H}_{2}$, which fall below $1100 \AA$ (e.g. Morton \& Dinerstein 1976), but it was not sensitive enough to observe PNe. IUE, the International Ultraviolet Explorer, observed many PNe at $\lambda \geqslant 1150 \AA$, including absorption-line studies of a few objects (i.e. NGC 6543 and BD+30 3639; Pwa, Pottasch, \& Mo 1984, 1986), but limited spectral resolution and dynamic range hampered attempts to extend this to a larger sample (Luhman \& Dinerstein 1990). A brief interlude of coverage below $1100 \AA$ was provided by HUT, the Hopkins Ultraviolet Telescope. Bowers et al. (1995) detected $\mathrm{H}_{2}$ in absorption towards NGC 1535, but were unable to distinguish between an IS or CS origin for the features due to HUT's low spectral resolving power.

Recently, the primary instruments for space UV spectroscopy have been GHRS (the Goddard High Resolution Spectrograph) and STIS (the Space Telescope Imaging Spectrograph) on $H S T$, both limited to $\lambda \geqslant 1160 \AA$. Observations with GHRS yielded many results on the diffuse ISM (Savage \& Sembach 1996), but relatively little on PNe. While $S T I S$ provided higher spectral resolution and more continuous spectral coverage than $G H R S$, it covers only one or a few multiplets, many of which are saturated, for most species (Williams et al. 2003). With its spectral range of $\sim 905-1180 \AA$, FUSE, the Far Ultraviolet Spectroscopic Explorer, covers the resonance lines of $\mathrm{H}_{2}$ as well as weaker (hence less saturated) transitions of many neutral and ionized species present in PNe. 


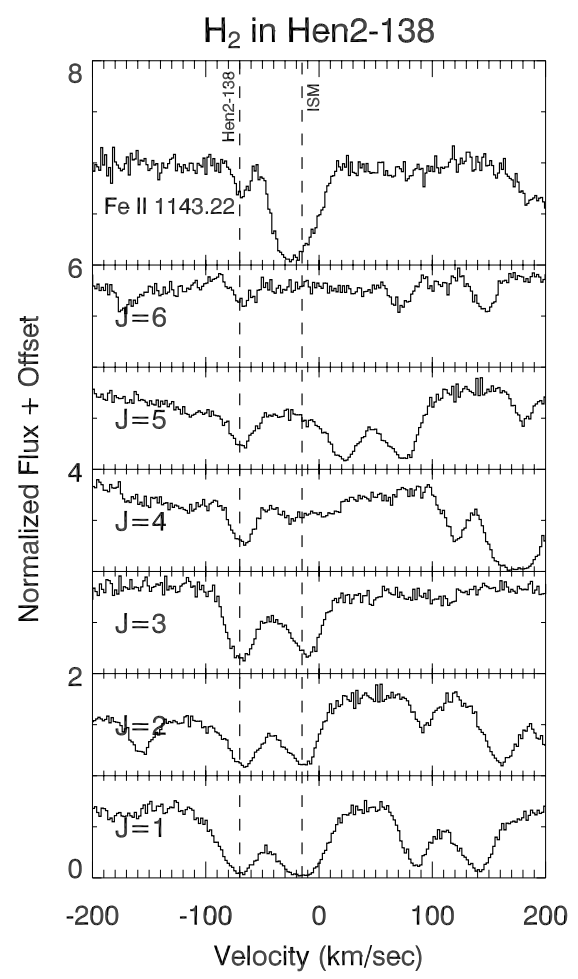

Figure 1. Velocity-resolved nebular $\mathrm{H}_{2}$ absorption features in the PN Hen 2-138 (Sonneborn \& Iping, in preparation). Note that features from higher $J$ levels remain prominent in the nebular component, but disappear from the interstellar system for $J \geqslant 4$.

\section{UV Observations of $\mathbf{H}_{2}$ in Planetary Nebulae}

It is a curious fact that $\mathrm{H}_{2}$, the most abundant molecule in the Universe, has essentially no spectroscopic signatures in the optical; virtually all observable spectral features lie in the infrared or ultraviolet. Apart from collisional excitation of the low rotation-vibration levels of the ground electronic state, all of the excited energy states of $\mathrm{H}_{2}$ are accessed primarily through radiative excitation (fluorescence). This begins with the absorption of a photon in the Lyman $\left(\mathrm{B}^{1} \Sigma_{u}{ }^{+}-\mathrm{X}{ }^{1} \Sigma_{g}{ }^{+}\right)$or Werner $\left(\mathrm{C}^{1} \Pi_{u}{ }^{+}-\mathrm{X}{ }^{1} \Sigma_{g}{ }^{+}\right)$bands at $\lambda \leqslant$ $1100 \AA$, followed by decay via a longer- $\lambda$ UV transition into the 14 bound $v$ states of the ground electronic state, or into the vibrational continuum where it dissociates (e.g. Black \& van Dishoeck 1987). This process populates the upper levels of the near-infrared lines of $\mathrm{H}_{2}$ seen in many PNe (e.g. Dinerstein et al. 1988; Likkel et al. 2006).

The initial absorption of a Lyman or Werner band photon can be seen with FUSE given the proper circumstances: a central star with a UV continuum sufficiently bright for obtaining high $S / N$ spectra; the presence of $\mathrm{H}_{2}$ along the line of sight; and a net radial velocity shift that separates the nebular absorption lines from those due to foreground material. An excellent example is shown in Fig. 1, which presents a sequence of profiles of lines arising from various $J$ levels for the PN He 2-138 (Iping et al. 1999; Sonneborn \& Iping, in preparation). It is immediately apparent that the nebular system exhibits higher excitation than the IS one, since the IS lines disappear for $J \geqslant 4$ while the nebular components persist to $J=6$. 
There have been several claimed detections of nebular $\mathrm{H}_{2}$ in Galactic and Magellanic Cloud (MC) PNe on the basis of high excitation alone, in objects where the nebular radial velocity is not well separated from that of the ISM (Herald \& Bianchi 2004a, b). These probably cannot be regarded as secure detections. Surveys of $\mathrm{H}_{2}$ in the ISM find that the presence of two distinct temperature components is common if not ubiquitous. While $T_{\text {rot }}$ values derived for the $J=1$ and 0 levels are $\approx 80-125 \mathrm{~K}$ in the Galactic ISM, the $J \geqslant 2$ lines require $T_{\text {rot }} \geqslant 200-500 K$ (Rachford et al. 2002; Gillmon et al. 2006). The excess population in the higher- $J$ levels is even more pronounced in the MCs, possibly due to a stronger mean IS UV radiation field (Tumlinson et al. 2002).

One of the most dramatic examples of nebular $\mathrm{H}_{2}$ in the UV spectrum of a CSPN is M27, the Dumbbell Nebula. The presence of hundreds of nebular $\mathrm{H}_{2}$ absorption lines in the FUSE spectrum of the Dumbbell was first reported by McCandliss et al. (2000). A detailed analysis finds that the nebular $\mathrm{H}_{2}$ has a low overall column density and an approximately (but not exactly) thermal population distribution with $T_{\text {exc }} \sim 2000 \mathrm{~K}$ (McCandliss et al. 2006, in preparation). The excitation appears not to be driven by the UV continuum fluorescence described above. However, line fluorescence is occurring in M27 (and also NGC 3132): nebular Lyman $\alpha$ photons are absorbed by $\mathrm{H}_{2}$ in $v=2$ levels, leading to a distinctive set of UV emission lines (Lupu, France, \& McCandliss, 2006).

These initial detections would seem to be encouraging with regard to the detectability of $\mathrm{H}_{2}$ in $\mathrm{PNe}$ in the UV. However, a targeted search for nebular $\mathrm{H}_{2}$ absorption lines in $\mathrm{PNe}$ with favorable radial velocities and known near-infrared $\mathrm{H}_{2}$ emission (FUSE GO programs A085 and B069, P.I. Dinerstein), produced mostly non-detections. Of our seven targets, only one showed nebular $\mathrm{UV} \mathrm{H}_{2}$ absorption (Dinerstein et al. 2006)! This apparent contradiction can be reconciled only if the $\mathrm{H}_{2}$ is confined to regions within the nebulae that are avoided by the UV absorption line of sight.

Consider the Ring and Dumbbell Nebulae: both show infrared $\mathrm{H}_{2}$ emission, and are believed to be intrinsically bipolar, with low-density lobes and a denser equatorial torus. Their different morphologies are caused by orientation effects: the Ring is nearly pole-on while the central star of the Dumbbell is viewed through the torus, where molecules are more likely to survive. Unlike the FUSE results for the Dumbbell, we set low upper limits on $\mathrm{N}\left(\mathrm{H}_{2}\right)$ in the Ring, $\sim 10^{-3}-10^{-4}$ times lower than the beam-averaged column density indicated by the IR lines. However, the IR line emission in the Ring arises from clumps in the main shell, none of which are projected along the sight-line to the central star (Speck et al. 2003). We find similar results for the compact PN SwSt 1, which has an irregular rather than bipolar morphology, but the central star is seen through a gap between clumps (De Marco et al. 2001). The lack of even trace amounts of $\mathrm{H}_{2}$ seen in absorption indicates that $\mathrm{H}_{2}$ is fully dissociated outside the clumps.

One of our targets, $\mathrm{BD}+30^{\circ} 3639$, represents a spectacular exception to this nondetection rule. We chose this target because of its striking nebular $\mathrm{Na}$ I lines in the optical (Dinerstein \& Sneden 1988). The FUSE spectrum of BD+30 is crowded by strong, often saturated IS components in the lower- $J$ transitions, but we also see $\mathrm{H}_{2}$ at the expected nebular velocity, as in He 2-138. A preliminary fit to the $v=0$ lines yields $T_{\text {rot }} \sim 800 \mathrm{~K}$ (Dinerstein \& Bowers 2004). In the STIS band (GO program 7283) the IS components conveniently vanish, leaving two nebular systems at $v_{\text {helio }}=-95$ and $-45 \mathrm{~km} \mathrm{~s}^{-1}$, seen in a plethora of levels up to $v=8$ and $J=11$ (Dinerstein \& Bowers et al., in preparation). Typical column densities are $\sim 10^{12-13} \mathrm{~cm}^{-2}$ per level, and $T_{v i b} \ggg T_{\text {rot }}$ for $J \geqslant 2$, consistent with radiative excitation. The ratio of ortho-to-para states is $\sim 1.7-2.5$ rather than the canonical (thermalized) value of 3 , another clear indicator of UV excitation (Black \& van Dishoeck 1987; Sternberg \& Neufeld 1999). 


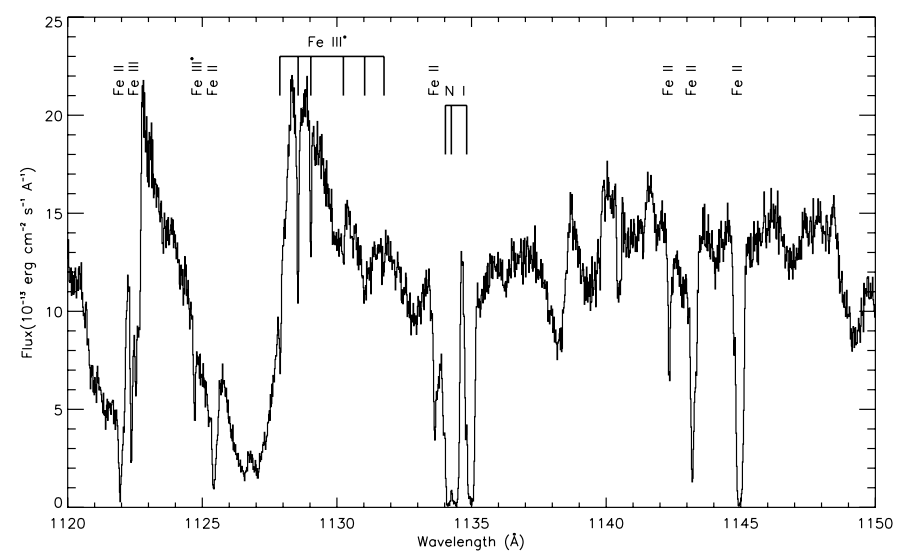

Figure 2. Section of the FUSE spectrum of SwSt 1, from SD05. The narrow lines, which include transitions of Fe II and Fe III, arise in the nebula or ISM. The broad lines with P Cygni profiles near the blue edge of this plot are due to the Wolf-Rayet type central star.

\section{UV Absorption Lines from the Ionized Gas}

As mentioned above, there have been relatively few UV absorption-line studies of the ionized gas in PNe. In this section, I will use a "case study" of the compact PN SwSt 1 (Sterling et al. 2005; hereafter SD05) to illustrate some of the kinds of information one can deduce from such observations. A segment of our spectrum is shown in Fig. 2.

While the UV absorption lines from the ground states of populous ions are often saturated, in some cases lines from the excited fine-structure levels are sufficiently weak that they can be used to estimate level-specific column densities. If this can be done for multiple excited fine-structure levels of an ion, for example the two excited levels of the ground ${ }^{3} P$ term of S III, the ratio of column densities for the two levels can be derived. If we assume that this is also the ratio of number densities, then we have a diagnostic tool, akin to the commonly-used temperature and/or density indicators based on optical and infrared emission line strength ratios. Since these are low-energy levels they have little dependence on $T_{e}$, but are quite sensitive $n_{e}$ indicators (e.g. Rubin 1989). From such an analysis of the S III multiplet at 1012-1021 $\AA$, SD05 found an electron density considerably lower than the value found from emission-line observations of this PN, and interpreted the difference to be caused by density variations within the nebula. (In view of this result, the assumption made by Williams et al. 2003 in a study of IC 418, that the relative populations of these same fine-structure levels are given by their LTE values the infinite-density case - is probably not justified.)

The ionic forms of Fe commonly found in PNe have ground configurations with a large number of low-lying energy levels, complicating the task of determining their gas-phase abundances. In the moderately low-excitation PN SwSt 1, the majority ion is $\mathrm{Fe}^{++}$, which has five fine-structure levels in its ground ${ }^{5} D$ term. Lines from all five of these levels are reported by SD05 in the FUSE spectrum of SwSt 1; however, we relied mainly on absorption lines from the second and third excited levels, in order to avoid saturation effects (which affect the ground and lowest excited levels) and measurement uncertainties (for very weak lines). For $\mathrm{Fe}^{+}$, only ground-state lines are detected, and the total column 
density is much lower than that of $\mathrm{Fe}^{++}$(a factor of 50 or more). Since there is no direct measure of $\mathrm{N}\left(\mathrm{H}^{+}\right)$in the pencil-beam column probed by the $U V$ absorption lines, we scaled our gas-phase column densities to $\mathrm{S}$, an element which is essentially unaffected by depletion into dust in the ISM (Savage \& Sembach 1996). This yielded an Fe/S ratio of about one-half to one-third solar, indicating only a mild depletion of Fe into dust. This is rather surprising, in view of the fact that emission lines from this PN in the optical (De Marco et al. 2001) and near-infrared (Likkel et al. 2006) indicate much more severe depletions of $\mathrm{Fe}$ in the gas phase, factors of 15 or more.

This disagreement between emission and absorption-line probes is reminiscent of our findings for $\mathrm{H}_{2}$ in SwSt 1 itself, as well as other PNe. However, in this case we are measuring in situ variations in the dust-to-gas ratio within a nebula. In view of the very young age of SwSt 1, estimated as $\sim 300$ years (De Marco et al.), it seems unlikely that either shock processing or photon sputtering can have effectively destroyed such a large fraction of the dust. Alternatively, these inhomogeneities in the dust-to-gas ratio may have been imprinted earlier, as variations in local dust formation efficiency in the clumpy AGB star atmosphere or wind (e.g. Grosdidier, Acker, \& Moffat 2000). Such variations may enhance the survival of molecules in the dustier regions of the nebula, while the absence of dust in other regions may promote the rapid photo-dissociation of the $\mathrm{H}_{2}$ and deny it the opportunity to re-form on grain surfaces, leading to the observed drastic variations in molecular fraction (see discussion and references in $\S 6$ of SD05).

Finally, in certain cases UV absorption lines can be used to detect elements that are not observed in the emission line spectrum, either because of low fractional abundances or unfavorable energy-level structures for their prevalent ions, or both. As an example, we have the trans-iron element Ge, which can be self-enriched within a PN progenitor by $s$-process nucleosynthesis reactions (see Busso, Sterling, this conference). Previously detected only in its neutral or singly-ionized form in the ISM and damped Ly $\alpha$ systems, Ge was detected via a resonance line of Ge III at $1088 \AA$ in the FUSE spectra of four PNe by Sterling, Dinerstein, \& Bowers (2002), who found it to be enhanced relative to the solar Ge/S ratio. Subsequently, Sterling \& Dinerstein (2003) detected Ge III in IC 4776, at a strength consistent with an unenriched (solar) value. In Sterling et al. (2005), we examined dust depletion effects in SwSt 1, and were able to verify that the elemental Ge abundance is enhanced by a factor of seven in this source.

\section{Tracers of the Photodissociation (Interface) Region}

The transition region between the fully ionized $\left(\mathrm{H}^{+}\right)$and fully molecular $\left(\mathrm{H}_{2}\right)$ zones of a gaseous nebula illuminated by a stellar source of UV radiation is often called a "PDR," standing for either "photo-dissociation region" or "photon-dominated region." Models of PDRs (Hollenbach \& Tielens 1999) are compared to observations, specifically the fluxes and ratios of the brightest cooling lines such as [C II] $158 \mu \mathrm{m}$, [O I] 63 and $145 \mu \mathrm{m}$, to infer parameters such as $n$, density, and $G_{o}$ or $\chi$, the intensity of the UV radiation field (Kaufman et al. 1999). However, it has been noted that the $145 \mu \mathrm{m}$ line often tends to be brighter than the models predict (e.g. Liseau et al. 1999; Mizutani et al. 2004).

The far-infrared [O I] lines arise from fine-structure levels of the ground ${ }^{3} P$ term. These levels also give rise to absorption lines in the UV. The strong multiplet at $1302-1306$ $\AA$, for example, was observable by both $I U E$ and $H S T$. Unsurprisingly, the ground-state transition (1302 $\AA$ ) is invariably blended with a very saturated corresponding IS feature. However, unfortunately the $1304 \AA$ line from the first excited level is also generally useless, due to an unhappy coincidence with a ground-state line of Si II. The $1306 \AA$ line is spectrally isolated, but still likely to be saturated (Fig. 3). 

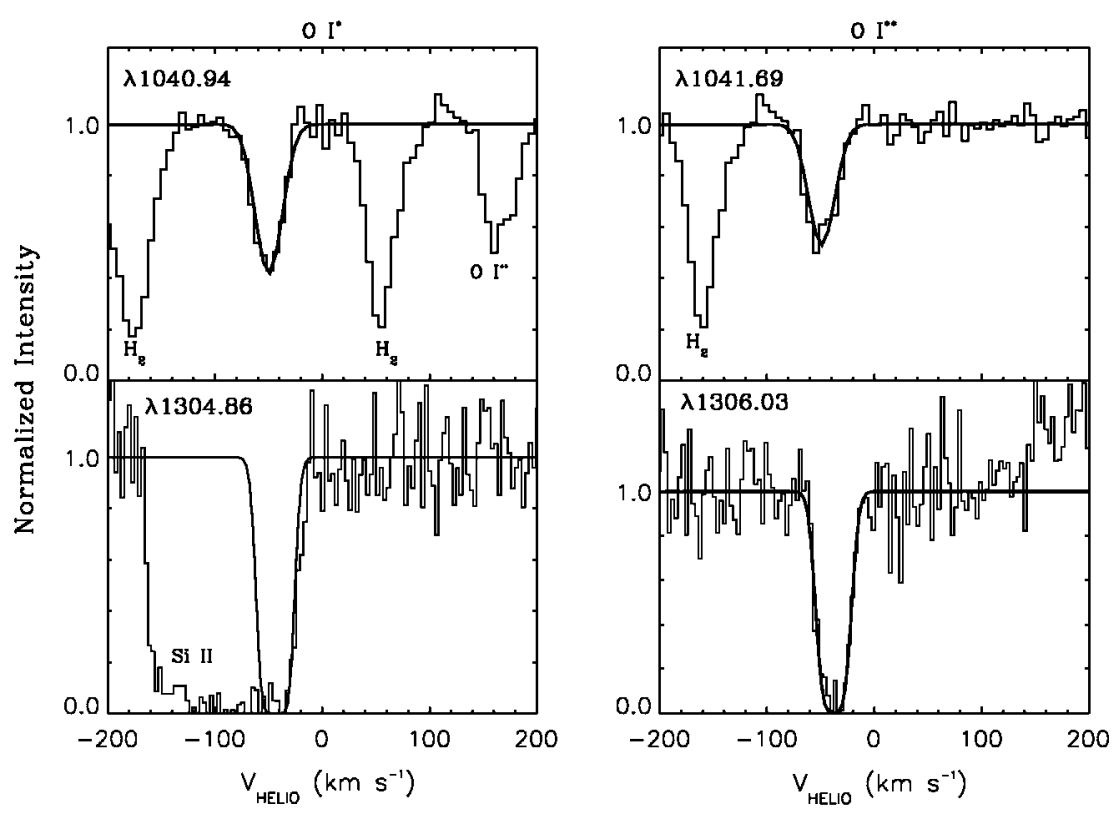

Figure 3. Absorption lines from the two excited fine-structure levels of O I, from SD05. The lines near $1041 \AA$ were observed with FUSE, the 1304, $1306 \AA$ lines with STIS. Both of the latter pair are highly saturated; O I* $1304.88 \AA$ also blends with a resonance line of Si II.

In contrast, the FUSE band contains a number of alternate $\mathrm{O}$ I multiplets, with a range of intrinsic strengths. Since the FUSE spectrum of SwSt 1 has low $S / N$ for $\lambda \leqslant$ $1000 \AA$, SD05 used the lines shown above. The column densities determined from 1040.94 and $1041.69 \AA$, which trace the originating levels of the 63 and $145 \mu \mathrm{m}$ lines respectively, indicates $n(J=0) / n(J=1)=0.7$, about twice the maximum possible ratio for pure collisional excitation. This population inversion clearly demonstrates that a non-collisional process is affecting the level populations. From the intensities of optical O I lines (De Marco et al.), SD05 concluded that this process is continuum fluorescence.

As discussed in $\S 4$, the line of sight towards the central star of SwSt 1 is not representative of the emission-line producing gas. However, it has enabled us to identify a mechanism which is likely to be relevant - though perhaps milder and/or diluted - in many other PDRs, since it results from the presence of a strong stellar radiation field, the defining property of a PDR. Failure to recognize the role of fluorescence can lead to misinterpretation of the far-infrared lines in terms of the true physical properties.

\section{Summary}

- UV absorption lines provide valuable new probes of nebular properties, such as $n_{e}$, $T_{e}$, and ionic and elemental abundances.

- UV $\mathrm{H}_{2}$ absorption is often absent in PNe with near-IR $\mathrm{H}_{2}$ emission. We infer that the molecular material is highly clumped and the UV line of sight often misses the clumps.

- Comparisons of absorption lines with emission lines of the same species reveals the presence of inhomogeneities in density, temperature, and local dust-to-gas ratio. This last effect may preserve and amplify the degree of clumping in $\mathrm{H}_{2}$.

- Continuum fluorescence can have a substantial effect on the fine-structure level populations of $\mathrm{O}$ I, with serious implications for some PDR diagnostic ratios. 


\section{Acknowledgements}

The author's research on PNe has been supported by FUSE support grants NAG 5-9239, 5-11597, 5-12731, and 5-13724, and STScI grant GO 07283.01-96A (for UV absorption-line work), and NSF grants 97-31156 and 04-06809. I also wish to thank G. Sonneborn and S. McCandliss for communicating results prior to publication.

\section{References}

Black, J., \& van Dishoeck, E.F. 1987, ApJ, 322, 412

Bowers, C.W., Blair, W.P., Long, K.S., \& Davidsen, A.F. 1995, ApJ, 444, 748

De Marco, O., Crowther, P.A., Barlow, M.J., Clayton, G.C., \& de Koter, A. 2001, MNRAS, 328, 527

Dinerstein, H.L. 1995, in: R. Williams \& M Livio (eds.), The Analysis of Emission Lines (Cambridge: Cambridge U. Press), 134

Dinerstein, H.L., \& Bowers, C.W. 2004, in: M. Meixner et al. (eds.), Asymmetrical Planetary Nebulae III, ASPC 313 (San Francisco: ASP), 347

Dinerstein, H.L., Lester, D.F., Carr, J.S., \& Harvey, P.M. 1988, ApJ, 327, L27

Dinerstein, H.L., \& Sneden, C. 1988, ApJ, 335, L23

Dinerstein, H.L., Sterling, N.C., \& Bowers, C.W. 2006, in: G. Sonneborn, H. Moos, \& B.-G. Andersson (eds.), Astrophysics in the Far Ultraviolet: Five Years of Discovery with FUSE, ASPC 348 (San Francisco: ASP), 328

Gillmon, K., Shull, J.M., Tumlinson, J., \& Danforth, C. 2006, ApJ, 636, 891

Grosdidier, Y., Acker, A., \& Moffat, A.F.J. 2000, A\&\&A, 364, 597

Herald, J.E., \& Bianchi, L. 2004a, ApJ, 609, 378

Herald, J.E., \& Bianchi, L. 2004b, ApJ, 611, 294

Hollenbach, D.J., \& Tielens, A.G.G.M. 1999, Rev Mod Phys, 71, 173

Iping, R.C., et al. 1999, BAAS, 195.06211

Kaufman, M.J., Wolfire, M.G., Hollenbach, D.J., \& Luhman, M.L. 1999, ApJ, 527, 795

Likkel, L., Dinerstein, H.L., Lester, D.F., Kindt, A., \& Bartig, K. 2006, AJ, 131, 1515

Liseau, R., et al. 1999, A\& A, 344, 342

Luhman, M.L., \& Dinerstein, H.L. 1990, BAAS, 22, 817

Lupu, R.E., France, K., \& McCandliss, S.R. 2006, ApJ, 644, 981

McCandliss, S.R. et al. 2000, BAAS, 197.0611 (http://fuse.pha.jhu.edu/wpb/sci_m27.html)

Mizutani, M., Onaka, T., \& Shibai, H. 2004, A\& A, 423, 579

Morton, D.C., \& Dinerstein, H.L. 1976, ApJ, 204, 1

Pwa, T.H., Pottasch, S.R., \& Mo, J.E. 1984, A\&A, 139, L1

Pwa, T.H., Pottasch, S.R., \& Mo, J.E. 1986, $A \mathscr{E} A$, 164, 184

Rachford, B.L., et al. 2002, ApJ, 577, 221

Rubin, R.H. 1989, ApJS, 69, 897

Savage, B.D., \& Sembach, K.R. 1996, ARAA, 34, 279

Speck, A.K., Meixner, M., Jacoby, G.H., \& Knezek, P.M. 2003, PASP, 115, 170

Spitzer, L., Jr., \& Jenkins, E.B. 1975, ARAA, 13, 133

Sterling, N.C., \& Dinerstein, H.L. 2003, Rev. Mexicana AyA (Ser. de Conf.), 18, 133

Sterling, N.C., Dinerstein, H.L., \& Bowers, C.W. 2002, ApJ, 578, L55

Sterling, N.C., Dinerstein, H.L., Bowers, C.W., \& Redfield, S. 2005, ApJ, 625, 368 (SD05)

Sternberg, A., \& Neufeld, D.A. 1999, ApJ, 516, 371

Tumlinson, J., et al. 2002, ApJ, 566, 857

Williams, R., Jenkins, E.B., Baldwin, J.A., \& Sharpee, B. 2003, PASP, 115, 178 